\title{
A STUDY ON SUDDEN EXPANSION HYDRODYNAMIC PHENOMENA OCCURRING IN CYLINDRICAL PIPES
}

\author{
Arestak Sarukhanyan*, Garnik Vermishyan \\ National University of Architecture and Construction of Armenia \\ Yerevan, Armenia
}

${ }^{*}$ Corresponding author: asarukhanyan51@mail.ru

\begin{abstract}
Introduction: This paper studies the frequency with which hydrodynamic parameters change in the sudden expansion section of axisymmetric pressure flow, based on the boundary layer equations. Methods: The suggested method reveals the regularity of changes in the hydrodynamic parameters of the flow in the transitional area, making it possible to obtain a velocity profile in any cross-section under common initial and boundary conditions. Based on the general solutions, we studied the hydrodynamic processes occurring in the transitional area of the effective sudden cross-section expansion within the axisymmetric pressure movement, in the following cases: a) when the velocity is constant at any point of the inlet face; b) when the velocity is distributed along the inlet face according to the parabolic law. Our calculations were carried

out for different values of the expansion factor: $\alpha=\frac{a}{R}=0.3 ; 0.5 ; 0.7$. Results: Based on the results of the computer-aided experimental study, we obtained velocity diagrams along the length of the transitional area with constant and parabolic velocity distributions for fluid inflowing into the expanded section. We also determined the patterns of pressure distribution along the length of the relevant section.
\end{abstract}

\section{Keywords}

Transitional area, effective cross-section, sudden expansion, velocity, distribution.

\section{Introduction}

In transitional areas of the effective cross-section (cross-section input and output, sudden expansion and narrowing, etc.), rearrangement of the velocity field is accompanied by a change in hydrodynamic parameters. The theoretical aim of this study, focused on the transitional area, was to determine how regularly hydrodynamic parameters change along the pipe and to develop calculation methods for energy losses.

A number of theoretical and approximate calculation methods were developed for studying the hydrodynamic phenomena occurring in the transitional areas of the input section. Each calculation method is based on conclusions regarding the flow's nature, which are used in theoretical studies and result reviews. Such conclusions are often related to specific flow intervals; therefore, the results obtained have limited applicability. The first known study on flow velocity change patterns in the transitional area of a circular pipe input was carried out by Boussinesq (Boussinesq, 1891). The main goal of that study was to assess disturbances in the velocity profile based on the velocity profile in the stabilized area. It should be noted that the spread of the obtained results is essential, especially for input-adjacent sections of the pipe. Schiller, using the boundary layer theory principles, conducted research
(Loitsyansky, 1973) on velocity rearrangement in the transitional input area, and developed an appropriate calculation methodology (Schiller, 1936). The obtained results provide good, consistent data on the central sections of the pipe when $r \leq R$. As for near-wall sections, this is where considerable disturbances occur (Targ, 1951).

Schlichting suggested a more accurate method for calculating the plane-parallel motion of the transitional input area (Schlichting, 1974). This method has been used to carry out a numerical integration of boundary layer equations. In addition to the numerical technique, Schlichting (1934) also suggested an analytical method for assessing the transitional area. This method is based on the idea that the flow range is divided into: a) the central section, where velocities are constant, b) the nearwall sections, where velocities keep changing according to the boundary layer regularity. The obtained solutions make it possible to reveal the velocity distribution pattern for any cross-section of the input section's transitional area. Yemtsev (1978) made use of the velocity parabolic distribution pattern in the boundary layer and constant velocity condition at the core. He arrived at similar conclusions. As a result, he found solutions for the velocity and pressure change in the plane-parallel motion input section of the transitional area. 
Slyozkin (1955) and Targ (1951) studied the regularities in the hydrodynamic parameters' behavior in the transitional area of the pipe input section. By making successive approximations and simplifying the Navier-Stokes equations, they formulated the boundary problem. Analytical solutions to this problem allow for determining how regularly the velocity and pressure change. The results obtained are remarkably consistent with the findings of experimental investigations. Comparative analyses confirm the reliability of the results obtained. It should be noted that the aforementioned studies pertain only to the input sections of the pipe. However, velocity field changes take place not only in input sections but also in sections where the pipe's geometrical parameters change. Studies on regularities of changes in the flow's hydrodynamic parameters in the aforementioned sections are of considerable practical interest and importance.

A similar study was carried out by Chen (1973), where approximate solutions were found under cylindrical and plane-parallel isotherm laminar motion conditions, ensuring satisfactory accuracy of the results. Studies of hydrodynamic phenomena running in the input transitional area along fluid lines in the aforementioned section were carried out in a 3D environment by integration of the Navier-Stokes equations' finite elements (Young, 2016). The studies demonstrate velocity and pressure distribution curves. Comparative analysis of the results obtained via numerical method was provided in (Hornbeck, 1964). Studies carried out in the boundary layer of the input section, under fourth-power (four-step) velocity change conditions (Mohanty and Asthana, 1979), resulted in laws of velocity and pressure change. Studies on velocity and pressure change regularities in circular-section cylindrical pipes and under plane-parallel motion conditions followed the method developed in (Sparrow et al., 2004) for fluid lines of arbitrary section. The results obtained were subjected to comparative analysis.

Belyaev et al. (2015) developed a mathematical model for identifying the velocity and pressure fields when viscous incompressible fluid flows in $2 D$ variable cross-section ducts in laminar flow mode. However, the solutions suggested are not applicable to determining the hydrodynamic parameters of the sudden expansion sections.

The studies listed above essentially present comments on phenomena occurring in the input section of the pipe. Meanwhile, hydrodynamic parameters' rearrangement processes also occur in other transitional areas. There are few papers dealing with this subject. By using the numerical integration of viscous-plastic fluid flow motion equations, researchers built flow lines and determined velocity and pressure changes along the axial direction within the sudden expansion section $(D / d=4)$ (Rocha et al., 2007). Mullin et al. (2009) carried out a thorough experimental investigation on the sudden expansion section. Using magnetic resonance imaging techniques, they successfully obtained quantitative estimates for velocity change in the transitional area. Hava and Rusak (2000) provided quantitative estimates for Navier-Stokes equations members under sudden symmetric and asymmetric expansion conditions. This results in the numerical integration of the nonlinear nonhomogeneous differential equations obtained by the researchers. Integration results were compared with experimentally obtained data. A number of important experimental investigations were conducted in the sudden expansion section (Fester et al., 2008). The researchers built a test rig and studied flows of both Newtonian and nonNewtonian fluids through sudden contractions of three diameter ratios of $0.22,0.5$, and 0.85 .

Sarukhanyan et al. (2020) reviewed the change patterns in the hydrodynamic parameters of viscous fluid laminar motion in the transitional area of the input section within a cylindrical pipe of $R$ radius under the initial arbitrary distribution of velocity conditions. Under such conditions, the viscous fluid is axisymmetric, and isotherm motion occurs. In the input section of the pipe, the velocity of fluid flow along the pipe walls, in accordance with the velocity diagram $u=\varphi(r)$, reaches zero, while the velocity diagram changes. These changes extend for a certain distance along the pipe. A boundary layer develops near the pipe walls. In the boundary layer, the velocity gradient $\frac{d u}{d n}$ becomes too large; for this reason, friction force values increase as well, regardless of the $\mu$ viscosity coefficient. The boundary layer gradually spreads and covers the entire pipe. Therefore, studies on the transitional area should use boundary layer equations.

Prandtl (Loitsyansky, 1973; Schlichting, 1974) suggested using the Navier-Stokes equations for the boundary layer.

Furthermore, Prandtl (Loitsyansky, 1973; Schlichting, 1974) obtained equations for the boundary layer by simplifying the Navier-Stokes equations. Viscous forces mostly act in the boundary layer. For this reason, while simplifying the Navier-Stokes equations, Prandtl neglected those equation members that were too small in comparison with viscous forces. He derived simplified equations for the boundary layer. This allowed for finding the regularities of viscous fluid laminar motion in the transitional area of the circular cylinder's input section.

\section{Theoretical Models}

We shall now study the hydrodynamic phenomena typical of viscous fluid laminar motion within the sudden expansion segment of a cylindrical pipe's section (Figure 1). The pipe is considered rigid; the fluid, incompressible.

The sudden expansion of a circular cylindrical pipe causes fluid velocity field deformation. Our study 

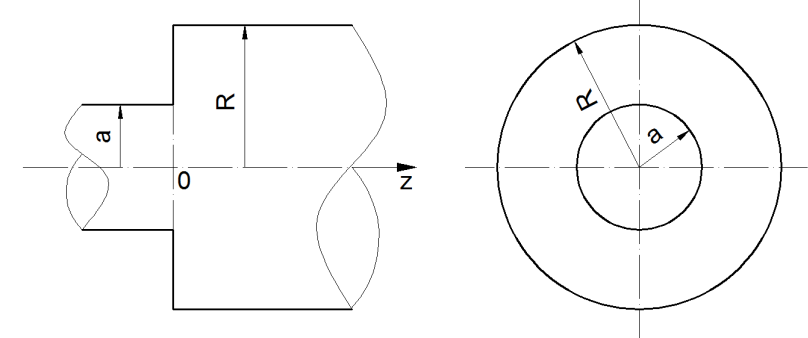

Figure 1. Sudden expansion segment: a - radius before expansion, $\mathbf{R}$ - radius after expansion

will concern viscous fluid laminar motion regularities in the velocity rearrangement section, using the same methodology as in research on the transitional area of the pipe's input section (Schlichting, 1974; Slyozkin, 1955). We shall use the following boundary layer equations for the cylindrical coordinate system (Loitsyansky, 1973; Schlichting, 1934):

$$
\begin{gathered}
u \frac{\partial u}{\partial r}+u \frac{\partial u}{\partial z}=-\frac{1}{\rho} \frac{\partial P}{\partial z}+v\left(\frac{\partial^{2} u}{\partial r^{2}}+\frac{1}{r} \frac{\partial u}{\partial r}\right) . \\
\frac{\partial u}{\partial z}+\frac{1}{r} \frac{\partial(v \cdot r)}{\partial r}=0
\end{gathered}
$$

To simplify Eq. (1), we take into consideration the conclusion made in (Schlichting, 1934, 1974) according to which $v<<u$, hence $v=0$. We arrive at:

$$
u \frac{\partial u}{\partial z}=-\frac{1}{\rho} \frac{\partial P}{\partial z}+v\left(\frac{\partial^{2} u}{\partial r^{2}}+\frac{1}{r} \frac{\partial u}{\partial r}\right)
$$

where $u$ and $v$ are the components describing velocity in the direction of $z$ and $r$ coordinates.

To integrate nonlinear nonhomogeneous differential equations, we make the following assumption: the $u$ coefficient of $\frac{\partial u}{\partial z}$ member is replaced by the average velocity of the effective cross-section.

$$
u=u_{0}=\frac{2}{R^{2}} \int_{0}^{R} \phi(r) r d r
$$

where $u=\varphi(r)$ is the function of velocity distribution in fluid entering the sudden expansion section.

Following this assumption, the study of regular patterns in the change of the hydrodynamic parameters within the sudden expansion segment (Figure 1) is reduced to the integration of the following equations:

$$
\begin{gathered}
u_{0} \frac{\partial u}{\partial z}=-\frac{1}{\rho} \frac{\partial P}{\partial z}+v\left(\frac{\partial^{2} u}{\partial r^{2}}+\frac{1}{r} \frac{\partial u}{\partial r}\right) . \\
\frac{\partial u}{\partial z}+\frac{1}{r} \frac{\partial(v \cdot r)}{\partial r}=0 .
\end{gathered}
$$

in case of the following initial and boundary conditions:

$$
\begin{gathered}
u=0, v=0 \text {, when } r=a \quad z=0 . \\
u=\varphi(r) \text {, when } z=0, \quad 0 \leq r \leq a . \\
u \rightarrow u^{\prime}, \text { when } z \rightarrow \infty, \quad 0 \leq r \leq a .
\end{gathered}
$$

where $V^{\prime}$ is the velocity of fluid in the stabilized section of the pipe, which is determined by the following equation:

$$
\frac{1}{\rho} \frac{\partial P}{\partial z}=v\left(\frac{\partial^{2} u^{\prime}}{\partial r^{2}}+\frac{1}{r} \frac{\partial u^{\prime}}{\partial r}\right)
$$

Eq. (10) has the following solution:

$$
u^{\prime}=-\frac{1}{\rho} \frac{\partial P}{\partial z} \frac{R^{2}}{4 v}\left(1-\frac{r^{2}}{R^{2}}\right)=2 u_{0}\left(1-\frac{r^{2}}{R^{2}}\right)
$$

where $u_{0}=\frac{V_{\max }^{\prime}}{2}=-\frac{1}{\rho} \cdot \frac{\partial P}{\partial z} \cdot \frac{R^{2}}{8 v}$ is the average velocity of the effective cross-section.

It follows from Eqs. (5) and (6) that, in each fixed section of the transitional area, $\frac{\partial P}{\partial r}=0$. Therefore, pressure in all points of the effective cross-section is equal and only changes when passing from one section to another $P=P(z)$.

Solution of Eq. (5) in case of (7), (8), (9) boundary conditions is Sarukhanyan et al (2020).

$$
\begin{gathered}
u(z, r)=-\sum_{k=1}^{\infty} C_{k}\left[J_{0}\left(\lambda_{k} \frac{r}{R}\right)-\frac{2 J_{1}\left(\lambda_{k}\right)}{\lambda_{k}}\right] \\
\exp \left(-\frac{\lambda_{k}^{2}}{R \cdot \operatorname{Re}} z\right)+2 u_{0}\left(1-\frac{r^{2}}{R^{2}}\right) \\
0 \leq r<R
\end{gathered}
$$

where $J_{0}\left(\lambda_{k}\right)$ is the zero-order Bessel function of the first kind, $J_{l}\left(\lambda_{k}\right)$ is the first-order Bessel function of the first kind, $J_{2}\left(\lambda_{k}\right)$ is the second-order Bessel function of the first kind. The eigenvalues of the problem are determined from the boundary condition, when $r=R$, $u=0$. As a result, we obtain 


$$
J_{0}\left(\lambda_{k}\right)-\frac{2 J_{1}\left(\lambda_{k}\right)}{\lambda_{k}}=0 \quad \text { or } \quad J_{2}\left(\lambda_{k}\right)=0
$$

which means that the eigenvalues of the problem are the roots of the second-order Bessel function of the first kind. We use the boundary condition for determining the values of $C_{K}$ constant'coefficients (8) and obtain the following:

$$
\begin{gathered}
\varphi(r)=-\sum_{k=1}^{\infty} C_{k} J_{0}\left(\lambda_{k}\right)\left[\frac{J_{0}\left(\lambda_{k} \frac{r}{R}\right)}{J_{0}\left(\lambda_{k}\right)}-1\right]+ \\
2 u_{0}\left(1-\frac{r^{2}}{R^{2}}\right) .
\end{gathered}
$$

We shall now multiply the two sides of Eq. (13) by orthogonal functions equivalent to eigenfunctions:

$$
\Phi_{n}\left(\lambda_{n} \frac{r}{R}\right)=\frac{8}{J_{0}\left(\lambda_{k}\right)}\left[\frac{J_{0}\left(\lambda_{k} \frac{r}{R}\right)}{J_{0}\left(\lambda_{k}\right)}-1\right]
$$

and after integration in the $0 \leq r<R$ interval, we obtain $C_{k}$ :

$$
\begin{gathered}
C_{k}=-\frac{8}{R^{2} J_{0}\left(\lambda_{k}\right)} \\
\int_{0}^{R}\left[\varphi(r)-2 u_{0}\left(1-\frac{r^{2}}{R^{2}}\right)\right]\left[\frac{J_{0}\left(\lambda_{k} \frac{r}{R}\right)}{J_{0}\left(\lambda_{k}\right)}-1\right] r \cdot d r .
\end{gathered}
$$

Let us represent Eq. (15) in the following form:

$$
C_{k}=-\frac{8}{R^{2} J_{0}\left(\lambda_{k}\right)}\left[L_{1}+\frac{u_{0} R^{2}}{2}\right]
$$

where

$$
L_{1}=\int_{0}^{R}\left[\frac{J_{0}\left(\lambda_{k} \frac{r}{R}\right)}{J_{0}\left(\lambda_{k}\right)}-1\right] \varphi(r) \cdot r \cdot d r .
$$

Pressure change behavior along the pipe is obtained from Eq. (5), while velocity change is obtained from Eq. (12), which results in the following equation:

$$
-\frac{1}{\rho} \cdot \frac{\partial P}{\partial z}=-\sum_{k=1}^{\infty} \lambda_{k}^{2} C_{k}\left[\begin{array}{c}
\frac{v}{R^{2}} J_{0}\left(\lambda_{k} \frac{r}{R}\right)- \\
\frac{u_{0}}{R \cdot \operatorname{Re}}\left[J_{0}\left(\lambda_{k} \frac{r}{R}\right)-\frac{2 J_{1}\left(\lambda_{k}\right)}{\lambda_{k}}\right] \\
\exp \left(-\frac{\lambda_{k}^{2}}{R \cdot \operatorname{Re}} z\right)
\end{array}\right]+\frac{8 u_{0} v}{R^{2}} .
$$

It follows that in the boundary state when $z \rightarrow \infty$ in case of pressure distribution and laminar motion, pressure change regularities coincide:

$$
\left(-\frac{\partial P}{\partial z}\right)_{z \rightarrow \infty}=\frac{8 \rho u_{0} v}{R^{2}}
$$

By integrating Eq. (15), we determine the regularity of pressure change:

$$
\begin{aligned}
& \frac{P_{0}-P}{\rho}=-\sum_{k=1}^{\infty} C_{k}\left[\begin{array}{c}
\frac{v \operatorname{Re}}{R} J_{0}\left(\lambda_{k} \frac{r}{R}\right)- \\
u_{0}\left[J_{0}\left(\lambda_{k} \frac{r}{R}\right)-\frac{2 J_{1}\left(\lambda_{k}\right)}{\lambda_{k}}\right]
\end{array}\right]+\frac{8 u_{0} v}{R^{2}} z . \\
& \exp \left(-\frac{\lambda_{k}^{2}}{R \cdot \operatorname{Re}} z\right)
\end{aligned}
$$

It follows from Eq. (20) that $P_{0}=P$ when $z=0$.

\section{Results and Discussion}

The solutions obtained are applicable to the general boundary and the initial conditions of the problem. By using general solutions, we can obtain new solutions, equivalent to the conditions provided for each special case. Let us now consider two special cases.

Case I. Let us assume that the velocity of fluid entering the sudden expansion section is constant at

all points. Thus, we have $\varphi(r)=u_{0}^{*}=$ const, $0 \leq r<a$, and define the value of $L_{1}$ accordingly:

$$
\begin{gathered}
L_{1}=\int_{0}^{a} u_{0}^{*}\left[\frac{J_{0}\left(\lambda_{k} \frac{r}{R}\right)}{J_{0}\left(\lambda_{k}\right)}-1\right] \cdot r \cdot d r= \\
u_{0}^{*}\left[\frac{R \cdot \alpha J_{1}\left(\lambda_{k} \frac{a}{R}\right)}{\lambda_{k} J_{0}\left(\lambda_{k}\right)}-\frac{a^{2}}{2}\right] .
\end{gathered}
$$

By substituting $L_{1}$ in Eq. (16) and taking into consideration that $u_{0}=\frac{u_{0}^{*} a^{2}}{R^{2}}$, we obtain the values
of $C_{k}$ coefficients:

$$
C_{k}=-\frac{8 u_{0} J_{1}\left(\alpha \lambda_{k}\right)}{\alpha \lambda_{k} J_{0}^{2}\left(\lambda_{k}\right)}
$$

Using the values of $C_{k}$ coefficients that we obtained, we determine the dimensionless regularity of the velocity change in the transitional area: 


$$
\begin{gathered}
\bar{u}=\frac{8}{\alpha} \sum_{k=1}^{\infty} \frac{J_{1}\left(\lambda_{k} \alpha\right)}{\lambda_{k} J_{0}\left(\lambda_{k}\right)}\left[\frac{J_{0}\left(\lambda_{k} \bar{r}\right)}{J_{0}\left(\lambda_{k}\right)}-1\right] \\
\exp \left(-\lambda_{k}^{2} \bar{z}\right)+2\left(1-\bar{r}^{2}\right),
\end{gathered}
$$

where $\bar{u}=\frac{u}{u_{0}}, \quad \alpha=\frac{a}{R}, \quad \bar{r}=\frac{r}{R}, \quad \bar{z}=\frac{z}{R \cdot \operatorname{Re}}$ are the dimensionless values.

To plot the diagrams of velocity distribution in case of the transitional area's sudden cross-sectional expansion, when the velocity of fluid entering the sudden expansion section is constant, we carried out numerical calculations for $a=0.3 ; 0.5 ; 0.7$ cases, using Eq. (23). Figure 2 presents combined diagrams of various $(\bar{z})$ velocity distribution patterns in different sections of the transitional area, and Figure 3 shows the same velocity change curves in different sections when $a=0.7$. The resulting graphs reveal the hydrodynamic picture of the processes occurring in the transitional area, which is important for the correct design of various hydraulic systems.

The plotted diagrams of velocity distribution in the transitional area enable the conclusion that the velocity diagram of fluid entering the sudden expansion section with a constant velocity undergoes a sudden change. Specifically, velocity increases in the center and decreases near the walls. Moreover, a motion of fluid in the opposite direction can be observed as well. This pattern, which starts at a certain $\bar{z}=0.2$ velocity in the direction of the motion, subsequently weakens, and at the end of the transitional area, we arrive at the parabolic distribution of the velocity.

Figure 4 shows graphs $\bar{u}=f(\bar{z})$ of velocity change along the length of the pipe at

$$
\bar{r}=\frac{r}{R}=0 ; 0.2 ; 0.4 ; 0.6 ; 0.8 ; 0.9
$$

points of the effective cross-section, for the $a=0.7$ case. It follows from the diagram of velocity rearrangement along the length of the pipe that in the cross-section of the sudden expansion entrance segment, the pattern of constant velocity distribution $u_{0}{ }^{*}=$ const becomes deformed, and velocity gets rearranged in the direction of the motion. At the end of the transitional area, the pattern of the velocity change becomes parabolic.

From the curves obtained, we derive the stabilization length. Given that in $\bar{r}=0$, the velocity equals $\bar{u}=0.99$, we obtain $\bar{z}=0.174$.

By substituting the values of $C_{k}$ coefficients from Eq. (22) in Eq. (20), we arrive at the pressure distribution function for the sudden expansion section.

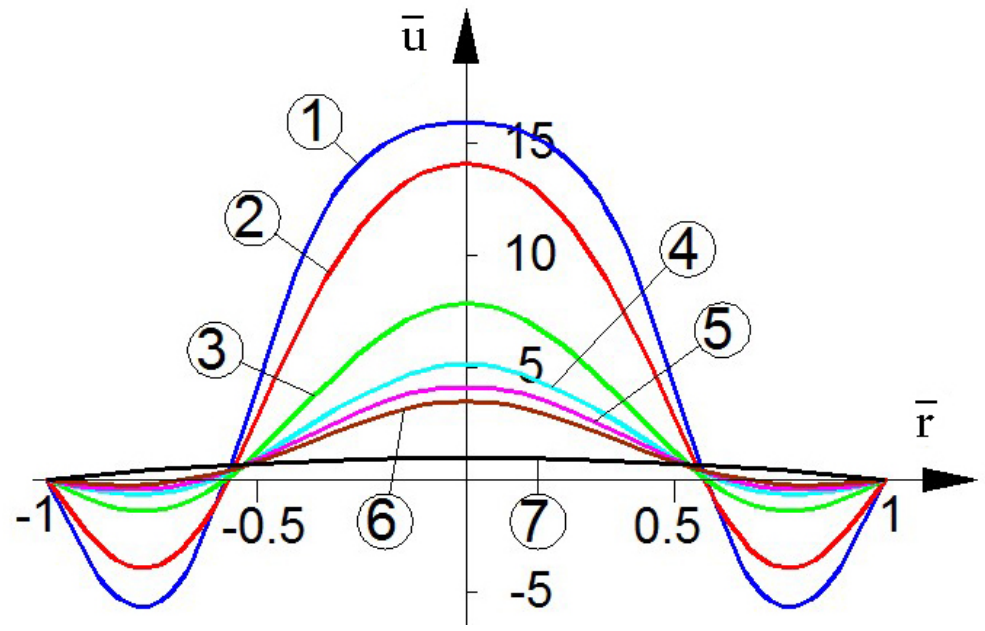

Figure 2. Superimposed graphs of lengthwise velocity change in the transitional area:

1. $\bar{z}=0.02 ; 2 . \bar{z}=0.03 ; 3 . \bar{z}=0.04 ; 4 . \bar{z}=0.06 ; 5 . \bar{z}=0.08 ; 6 . \bar{z}=0.09 ; 7 . \bar{z}=0.2$

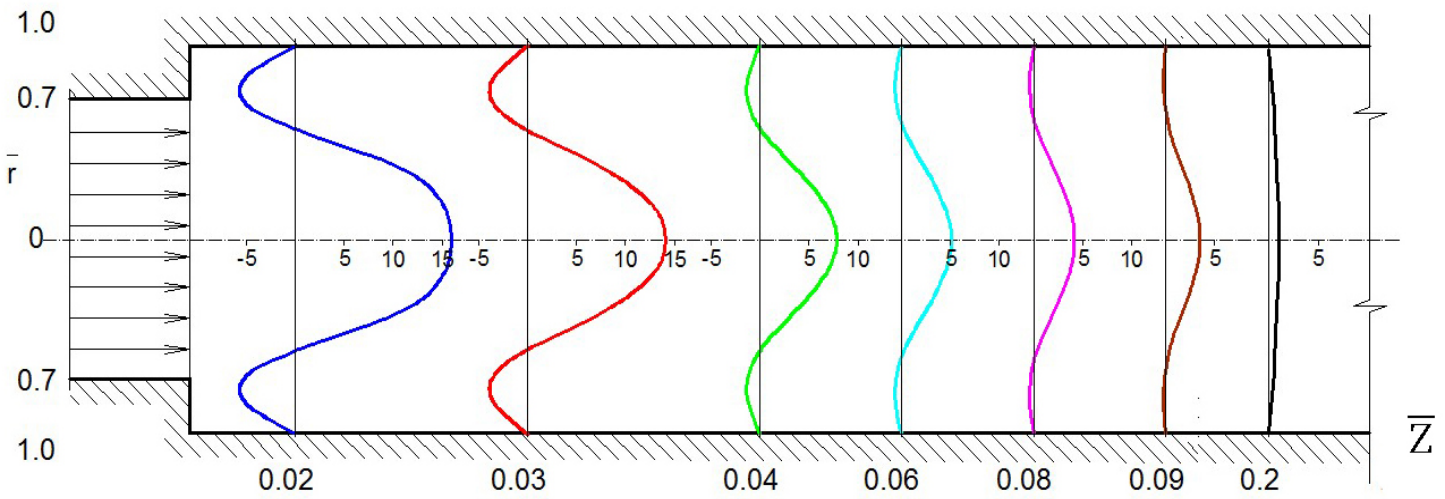

Figure 3. Graphs of lengthwise velocity change in the transitional area depending on $\bar{z}$ and $\bar{r}$ coordinates 


$$
\frac{P_{0}-P}{\rho}=8 u_{0} \sum_{k=1}^{\infty} \frac{J_{1}\left(\alpha \lambda_{k}\right)}{\lambda_{k} J_{0}^{2}\left(\lambda_{k}\right)}\left[\left[\left(\frac{v \mathrm{Re}}{R}-u_{0}\right) J_{0}\left(\lambda_{k} \frac{r}{R}\right)+u_{0} J_{0}\left(\lambda_{k}\right)\right]\left(1-\exp \left(-\frac{\lambda_{k}^{2}}{R \cdot \operatorname{Re}} z\right)\right)\right]+\frac{8 u_{0} v}{R^{2}} z
$$

Case II. Let us assume that the velocity distribution of fluid entering the sudden expansion section is parabolic. Therefore,

we have $\varphi(r)=2 u_{0}^{*}\left(1-\frac{r^{2}}{a^{2}}\right), \quad 0 \leq r<a$. Accordingly, the value of $L_{1}$ is defined as follows:

$$
\begin{aligned}
L_{1}=2 u_{0}^{*} \int_{0}^{a}\left[\frac{J_{0}\left(\lambda_{k} \frac{r}{R}\right)}{J_{0}\left(\lambda_{k}\right)}-1\right]\left(1-\frac{r^{2}}{a^{2}}\right) \cdot r \cdot d r= \\
2 u_{0}^{*}\left[\frac{2 R^{2} J_{2}\left(\lambda_{k} \alpha\right)}{\lambda_{k}^{2} J_{0}\left(\lambda_{k}\right)}-\frac{a^{2}}{4}\right] .
\end{aligned}
$$

As we substitute $L_{1}$ from Eq. (24) into Eq. (16) and take $u_{0}=\frac{u_{0}^{*} a^{2}}{R^{2}}$ into account, we obtain the coefficient $C_{k}$

$$
C_{k}=-\frac{32 u_{0} J_{2}\left(\lambda_{k} \alpha\right)}{\alpha^{2} \lambda_{k}^{2} J_{0}^{2}\left(\lambda_{k}\right)}
$$

Having obtained the values of $C_{k}$ coefficients, we arrive at the dimensionless patterns for the velocity change in the transitional area:

$$
\begin{gathered}
\bar{u}=\frac{32}{\alpha^{2}} \sum_{k=1}^{\infty} \frac{J_{2}\left(\lambda_{k} \alpha\right)}{\lambda_{k}^{2} J_{0}\left(\lambda_{k}\right)}\left[\frac{J_{0}\left(\lambda_{k} \bar{r}\right)}{J_{0}\left(\lambda_{k}\right)}-1\right] \\
\exp \left(-\lambda_{k}^{2} \bar{z}\right)+2\left(1-\bar{r}^{2}\right) .
\end{gathered}
$$

To discover velocity change patterns for sudden cross-sectional expansion in the transitional area,

we have made calculations for $\alpha=0.3 ; 0.5 ; 0.7$ cases, based on Eq. (26). On the basis of the values obtained, we have plotted diagrams representing the general behavior of velocity distribution in different cross-sections of the transitional area in Figure 5. Figure 6, in turn, shows separate diagrams according to the cross-sections $(\bar{z})$ in case $\alpha=0.5$.

Figure 7 shows graphs $\bar{u}=f(\bar{z})$ of velocity change along the length of the pipe at

$\bar{r}=0 ; 0.2 ; 0.4 ; 0.8 ; 0.9 \quad$ points of the effective cross-section, for the $\alpha=0.5$ case. It follows from the diagram of velocity rearrangement along the length of the pipe that in the cross-section of the sudden expansion entrance segment, the pattern of parabolic velocity distribution

$$
u=2 u_{0}^{*}\left(1-\frac{r^{2}}{a^{2}}\right)
$$

becomes deformed, and velocity gets rearranged in the direction of the motion. At the end of the transitional area, the pattern of the velocity change becomes parabolic.

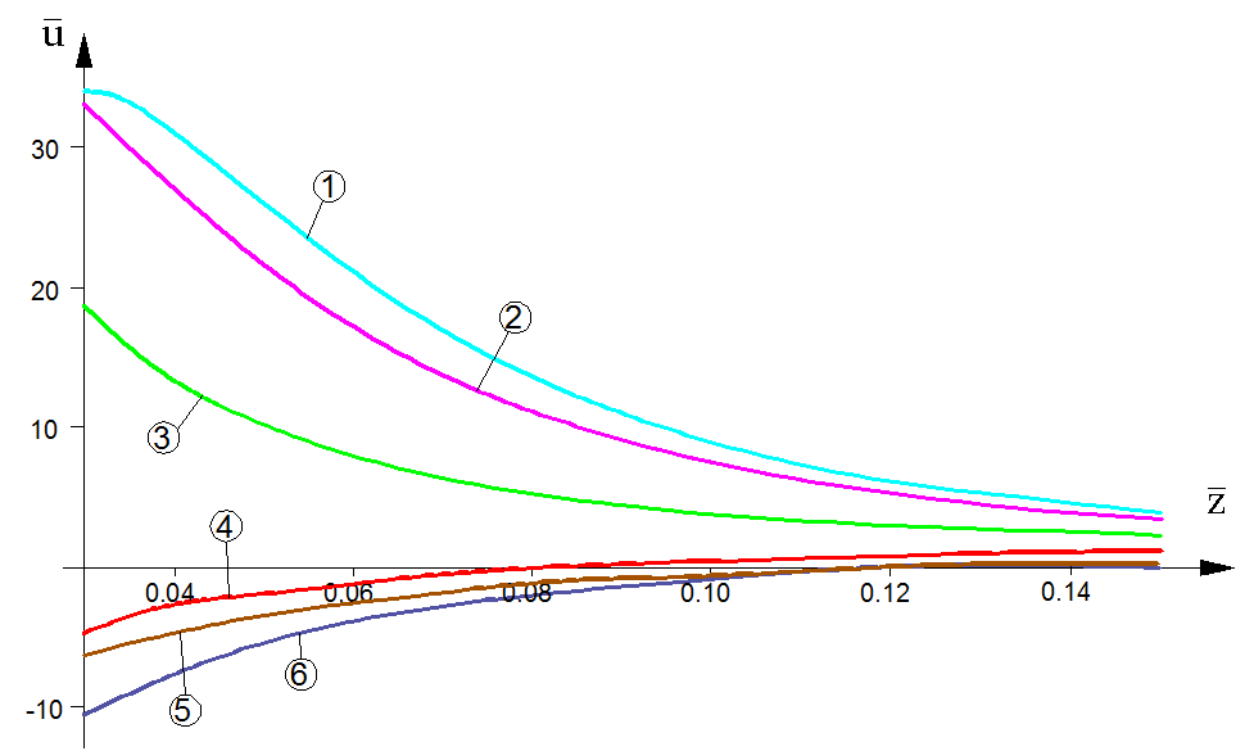

Figure 4 . Velocity change in $\bar{r}=0 ; 0.2 ; 0.4 ; 0.6 ; 0.8 ; 0.9$ points of the effective cross-section: 1. $\bar{r}=0 ;$ 2. $\bar{r}=0.2 ;$ 3. $\bar{r}=0.4: 4 . \bar{r}=0.6 ; 5 . \bar{r}=0.8 ; 6 . \bar{r}=0.9$. 


$$
\frac{P_{0}-P}{\rho}=32 \frac{u_{0}}{\alpha^{2}} \sum_{k=1}^{\infty} \frac{J_{2}\left(\alpha \lambda_{k}\right)}{\lambda_{k}^{2} J_{0}^{2}\left(\lambda_{k}\right)}\left[\left(\frac{v \operatorname{Re}}{R}-u_{0}\right) J_{0}\left(\lambda_{k} \frac{r}{R}\right)+u_{0} J_{0}\left(\lambda_{k}\right)\left(1-\exp \left(-\frac{\lambda_{k}^{2}}{R \cdot \operatorname{Re}} z\right)\right)\right]+\frac{8 u_{0} v}{R^{2}} z
$$

According to the graphs provided in the figures above, the length of the stabilization section is obtained under the following conditions: when $\bar{r}=0$ the velocity equals, $\frac{u}{u_{0}}=0.99$ we obtain $\bar{z}=0.163$.

To obtain the pressure distribution function in the sudden expansion section, we substitute the values of $C_{k}$ coefficient from Eq. (25) into Eq. (20), and arrive at the following.

\section{Conclusion}

The proposed universal method, designed for calculating velocity rearrangement in the transitional area, makes it possible to find the regular patterns in hydrodynamic parameter changes under general boundary conditions. The use of the relations that we discovered has defined the process of velocity field changes during sudden cross-sectional expansion, provided that the presence of fluid is constant and the parabolic distribution law conditions are met. This makes it possible to calculate the changes in the hydrodynamic flow parameters, as well as make generalizations. The regularities characterizing the velocity field changes in the transitional area, along with the diagrams that illustrate them, allow for correcting the design of the relevant hydromechanical equipment units.

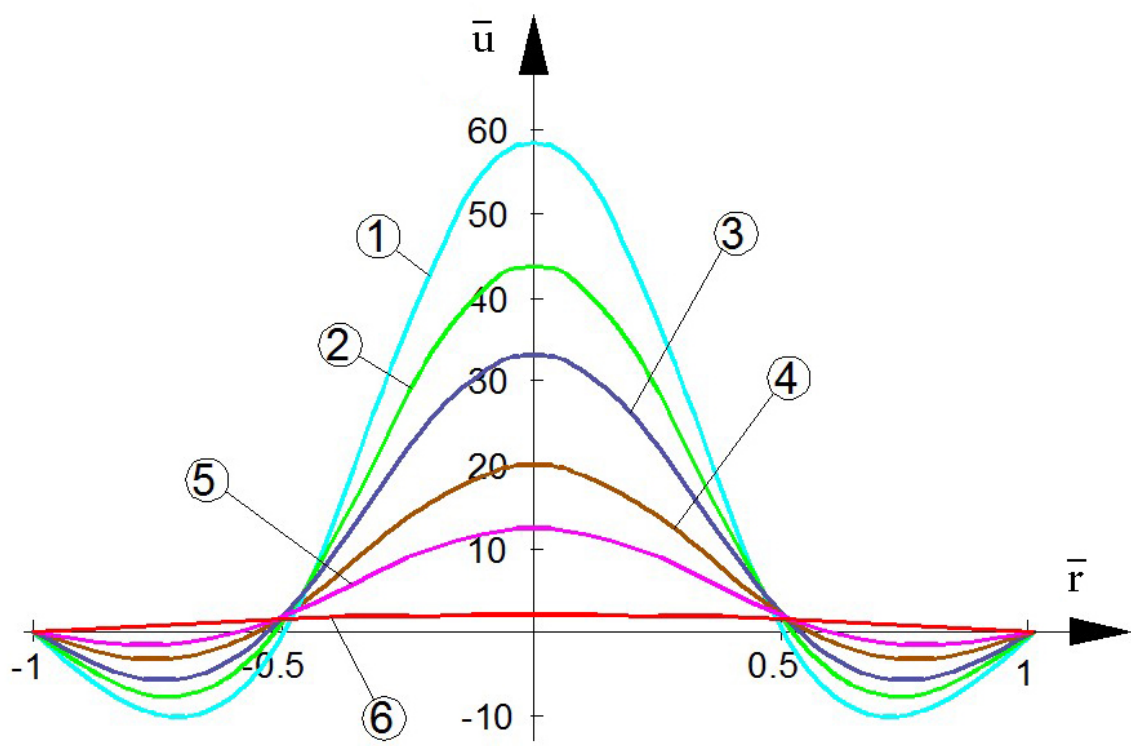

Figure 5. Superimposed graphs of lengthwise velocity change in the transitional area: 1. $\bar{z}=0.04 ; 2 . \bar{z}=0.05 ; 3 . \bar{z}=0.06 ; 4 . \bar{z}=0.08 ; 5 . \bar{z}=0.1 ; 6 . \bar{z}=0.2$

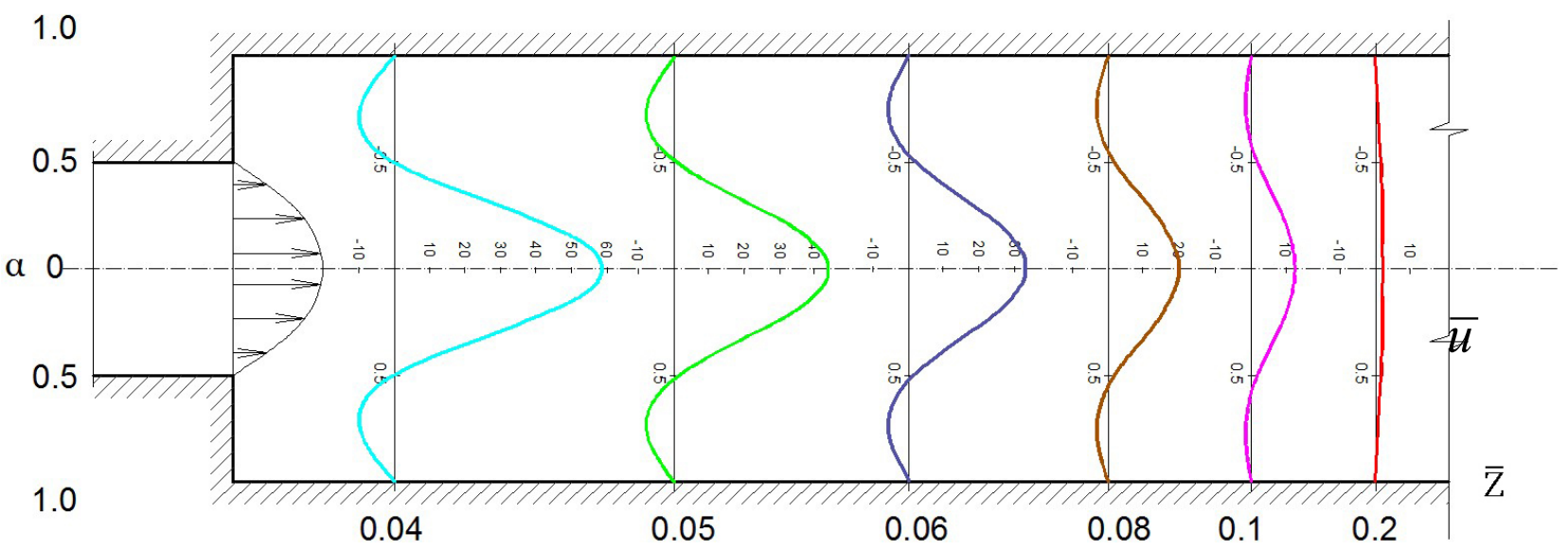

Figure 6. Curves of lengthwise velocity change in the transitional area depending on $\bar{Z}$ and $\bar{r}$ coordinates 


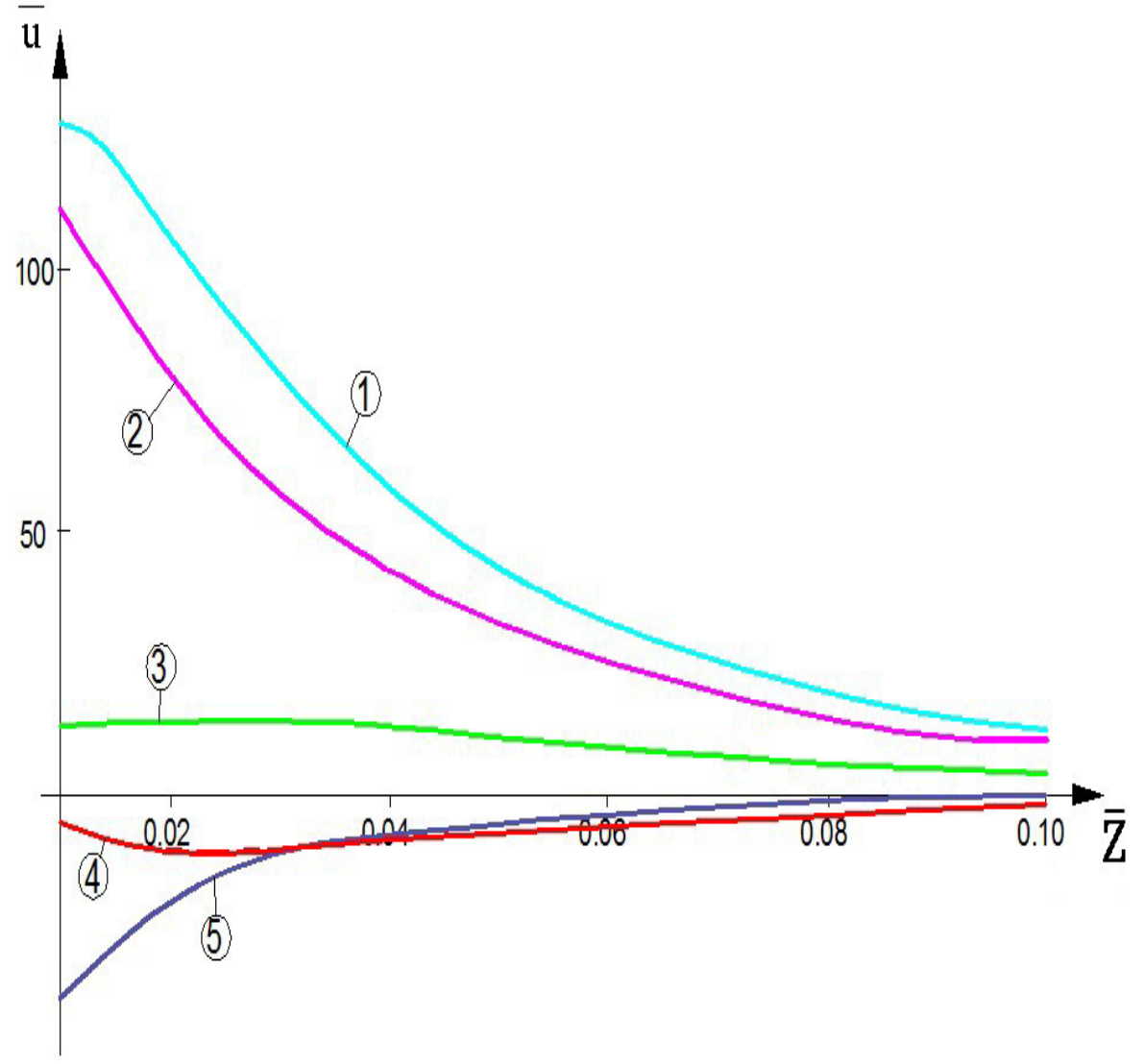

Figure 7. Velocity change in $\bar{r}=0 ; 0.4 ; 0.8 ; 0.9$ points of the effective cross-section: 1. $\bar{r}=0 ; 2 . \bar{r}=0.2 ;$ 3. $\bar{r}=0.4 ; 4 . \bar{r}=0.8 ; 5 . \bar{r}=0.9$ 


\section{References}

Belyaev, L. A., Zaitsev, A. S., Kondakov, A. A., Shevelev, S. A., Valkov, E. P. and Matveeva, A. A. (2015). Numerical analysis of fluid particles motion in curved ducts. MATEC Web of Conferences, Vol. 37, 01007. DOI: 10.1051/ matecconf/20153701007.

Boussinesq, J. (1891). Comptes rendus hebdomadaires des séances de l'Académie des sciences, Vol. CXII (1), pp. 9-14, 49-51.

Chen, R.-Y. (1973). Flow in the entrance region at low Reynolds numbers. Journal of Fluids Engineering, Vol. 95, Issue 1, pp. 153-158. DOI: 10.1115/1.3446948.

Durst, F., Ray, S., Ünsal, B. and Bayoumi, O. A. (2005). The development lengths of laminar pipe and channel flows. Journal of Fluids Engineering, Vol. 127, Issue 6, pp. 1154-1160. DOI: 10.1115/1.2063088.

Fester, V., Mbiya, B. and Slatter, P. (2008). Energy losses of non-Newtonian fluids in sudden pipe contractions. Chemical Engineering Journal, Vol. 145, Issue 1, pp. 57-63. DOI: 10.1016/j.cej.2008.03.003.

Gücüyen, E., Erdem, R. T. and Gökkuş, Ü. (2019). Numerical modelling of sudden contraction in pipe flow. Sigma: Journal of Engineering and Natural Sciences, Vol. 37, No. 3, pp. 903-916.

Hava, T. and Rusak, Z. (2000). Viscous flow in a slightly asymmetric channel with a sudden expansion. Physics of Fluids, Vol. 12, Issue 9, pp. 2257-2267. DOI: 10.1063/1.1287610.

Hornbeck, R. W. (1964). Laminar flow in the entrance region of a pipe. Applied Scientific Research, Section A, Vol. 13, pp. 224-232. DOI: 10.1007/BF00382049.

Letelier, S. M. F. and Leutheusser, H. J. (1983). Unified approach to the solution of problems of unsteady flow in long pipes. Journal of Applied Mechanics, Vol. 50, Issue 1, pp. 8-12. DOI: 10.1115/1.3167023.

Loitsyansky, L.G. (1973). Fluid and gas mechanics. $4^{\text {th }}$ edition. Moscow: Nauka, 847 p.

Mohanty, A. K. and Asthana, S. B. L. (1979). Laminar flow in the entrance region of a smooth pipe. Journal of Fluid Mechanics, Vol. 90, Issue 3, pp. 433-447. DOI: 10.1017/S0022112079002330.

Mullin, T., Seddon, J. R. T., Mantle, M. D. and Sederman, A.J. (2009). Bifurcation phenomena in the flow through a sudden expansion in a circular pipe. Physics of Fluids, Vol. 21, Issue 1, 014110. DOI: 10.1063/1.3065482.

Rocha, G. N., Poole, R. J. and Oliveira, P. J. (2007) Bifurcation phenomena in viscoelastic flows through a symmetric 1:4 expansion. Journal of Non-Newtonian Fluid Mechanics, Vol. 141, Issue 1, pp. 1-17, DOI: 10.1016/j.jnnfm.2006.08.008.

Sarukhanyan, A., Vartanyan, A., Vermishyan, G. and Tokmajyan, V. (2020). The study of hydrodynamic processes occurring on transition of sudden expanding of hydraulic section of plane - parallel full pipe flow. TEM Journal, Vol. 9, Issue 4, pp. 1494-1501. DOI: 10.18421/TEM94-23.

Schiller, L. (1936). Fluid flow in pipes. Moscow - Leningrad: Joint Scientific and Technical Publishing House (ONTI).

Schlichting, H. (1934). Laminare Kanaleinlauf Strömung. ZAMM, No. 14, pp. 368-373.

Schlichting, H. (1974). Theory of boundary layer. Moscow: Nauka, 711 p.

Slyozkin, N. A. (1955). Dynamics of viscous incompressible fluid. Moscow: Gostekhizdat, 520 p.

Sparrow, E. M., Lin, S. H. and Lundgren, T. S. (2004). Flow development in the hydrodynamic entrance region of tubes and ducts. The Physics of Fluids, Vol. 7, Issue 3, 338. DOI: 10.1063/1.1711204.

Targ, S. M. (1951). Fundamental problems of the theory of laminar flow. Moscow: Gostekhizdat, 420 p.

Yemtsev, B. T. (1978). Technical hydrodynamics. Moscow: Mashinostroyeniye, 463 p.

Young, F. J. (2016). The entrance region of circular pipes revisited. Open Access Library Journal, Vol. 3, No. 7, pp. 1-7. DOI: 10.4236/oalib.1102675. 